\title{
Elementos teórico-metodológicos que sustentan el papel de investigación en la Maestría Profesional en Planificación de la Universidad Nacional
}

Theoretical-methodological elements substantiating the role of investigation in the Professional Master's Degree in Planning program of the Universidad Nacional

\author{
Daniel Láscarez Smith \\ Vocational Education and Training, Osnabrück University, Germany \\ dlascarez@utn.ac.cr
}

\section{Ángel Abelino Ortega}

Escuela de Planificación y Promoción Social, Universidad Nacional, Costa Rica angel.ortega.ortega@una.cr

Luis Fernando Morales-Abarca

Escuela de Planificación y Promoción Social, Universidad Nacional, Costa Rica luis.morales.abarca@una.cr

Recibido: 27/11/2019 • Aceptado: 14/05/2021

\begin{abstract}
Resumen
Una de las principales preocupaciones que expresan los estudiantes que cursan alguno de los dos énfasis de la Maestría Profesional de Planificación (MPP) ${ }^{1}$ está relacionada, por un lado, con las características administrativas y formativas que tienen las maestrías profesionales y las maestrías
\end{abstract}

1 Maestria en planificación estratégica y en formulación y gestión de proyectos, EPPS, UNA. 
académicas; por otro lado, os alcances y las formas que adquiere la investigación científica en el ámbito profesional de la planificación. Esto ha derivado en que, en los últimos años, las personas estudiantes de la maestría experimenten dificultades para plantear, de forma clara y exitosa, las propuestas de trabajos finales de graduación (TFG). Este artículo es el resultado de un proceso de investigación, realizado con las personas estudiantes, con las personas expertas curriculistas y con el cuerpo docente de la Maestría Profesional en Planificación, sobre la relevancia de la investigación científica en contextos profesionales de la Planificación. Mediante una metodología cualitativa, se obtuvieron importantes hallazgos acerca de las dificultades epistemológicas y técnicas que emergen al proponer una investigación en ámbitos técnicos y profesionales. Trabajar sobre estos hallazgos permite mejorar la orientación científica y pedagógica de los nuevos estudiantes en relación con la comprensión del objeto de estudio de la planificación en el ámbito profesional y cómo la investigación aplicada juega un papel fundamental para el análisis del contexto sociopolítico, económico e institucional.

Palabras clave: Planificación, Investigación, Proyecto de investigación, Maestría Profesional.

\begin{abstract}
One of the main concerns expressed by students who are studying one of the two specializations of the Professional Master's Degree in Planning (PMP) ${ }^{2}$ program is related, on the one hand, to the administrative and educational characteristics of the professional and academic master's degrees, and on the other hand, to the scope and forms of the scientific research required in professional planning. As a result, in recent years, candidates for the master's degree have experienced difficulties in clearly and successfully submitting their proposals for final graduation projects (FGP). This article presents the results of investigations on the relevance of scientific research in professional Planning contexts, carried out with students, curriculum experts and the faculty of the Professional Masters Degree in Planning (PMP). Using a qualitative methodology,
\end{abstract}

2 Master's Degree in Strategic Planning and in Project Design and Management, EPPS (School of Planning and Social Promotion), UNA. 
important findings were obtained about the epistemological and technical difficulties encountered when proposing research in technical-professional environments. These findings can assist in improving the scientific-educational orientation of new students with regard to understanding the object of studying professional planning, and how applied research plays a fundamental role in the analysis of socio-political, economic and institutional contexts.

Keywords: Planning, Research, Research projects, Professional Master's Degree

\section{Introducción}

La oferta de estudios de posgrado registrada por el CONARE en 2016, comprende un total de 469 opciones, de esa cifra, un 67\% corresponde a la oferta de posgrados en las universidades públicas, es decir, 314 opciones de estudio de posgrado. La Universidad de Costa Rica tenía registradas 210 opciones de posgrado, las otras 104 opciones de estudio eran ofrecidas entre la Universidad Nacional, la Universidad Estatal a Distancia, el Instituto Tecnológico de Costa Rica y la Universidad Técnica Nacional, según datos publicados en el sexto informe del estado de la educación del Programa Estado de la Nación (2017, p. 252). Esto ha permitido que las personas académicas y profesionales del ámbito institucional público, empresa privada, organizaciones no gubernamentales y las personas trabajadoras independientes encuentren diversas opciones de formación a nivel de posgrado según sus intereses profesionales y académicos particulares.

Además de los criterios que juegan un papel central como el de la calidad del programa, el prestigio del centro educativo, los costos, el financiamiento, la duración, su relación con el ámbito laboral y el tipo de modalidad (virtual, bimodal, presencial entre otros), una de las decisiones más importantes al momento de escoger entre uno y otro posgrado de maestría, es el tipo de orientación que posee el programa, ya sea académico sea profesional, pues en este último punto se definen muchos de los aspectos generales y particulares de la maestría, por ejemplo, la estructura a nivel curricular del plan de estudios, distribución de créditos, la carga académica dedicada a los fundamentos investigativos, el tipo de trabajo final de graduación, las formas de mediación pedagógica, y el tipo de título que 
certifica la conclusión de los estudios (como Msc. MA, Magister, MB, entre otros.), entre otros.

En términos generales, se puede decir que la oferta de maestrías cuyo enfoque está más relacionado con obtener competencias de alto nivel teórico y práctico, las cuales nacen de las necesidades institucionales públicas y privadas, tienen una orientación profesional (MP), mientras que las maestrías que pretenden que la persona estudiante obtenga competencias científicas de alto nivel para investigar complejos objetos de estudio a través del desarrollo de paradigmas, teorías, conceptos y categorías y métodos científicos, corresponden a las maestrías académicas (MA). Pero las preguntas que se deberían hacer son ¿cómo se gestionan y ejecutan las MA y MP en las dinámicas de aprendizaje dentro y fuera del aula, dadas las diferencias fundamentales que tienen? $Y$ ¿cómo se debe mediar el aprendizaje de los fundamentos de la investigación científica al tomar en cuenta esas diferencias básicas?, ¿acaso es que la investigación científica no es necesaria para entender los problemas organizacionales en las empresas privadas y en las instituciones públicas?

Detrás de estas preguntas se esconden ciertos problemas pertinentes para la discusión: (a) el problema pedagógico y de mediación sobre el aprendizaje de competencias investigativas que recae sobre el cuerpo docente; (b) el problema de la comprensión de la construcción del objeto de estudio de una investigación en el campo de la planificación; y (c) el problema del entendimiento del objeto disciplinar de la Planificación y la forma en que este entendimiento se relaciona con las necesidades profesionales y con los requerimientos formales de los TFG. La diferencia entre el objeto de estudio y el objeto disciplinar según Ríos y Quiroz Bañol (2018), es que:

Un objeto de estudio se asume como un problema específico al que se quiere buscar solución en el corto plazo, mientras que el objeto de estudio disciplinar está más referido a la fundamentación conceptual y filosófica de una disciplina con miras a favorecer su avance mediante la construcción del conocimiento científico (p. 3).

Los objetos de investigación consisten en "una construcción del investigador, intermedia entre sus presupuestos teóricos y las referencias empíricas de la realidad" (Torres Carrillo \& Jiménez Becerra, 2004, p. 26). 
Comprender el proceso de construcción y de fundamentación de objetos de estudio en la Planificación permitiría redefinir la estrategia administrativa y académica para acompañar adecuadamente al estudiantado desde el primer día de clases en la problematización científica de los contextos profesionales de la Planificación. Es decir, ya no se trataría solamente de preguntar ¿cómo se formula un "buen" proyecto socioeconómico o un "buen" plan estratégico?, ¿cuáles son los problemas de planificación que enfrentan las instituciones y empresas específicas para la consecución de sus metas y objetivos?, ¿cómo se añade valor público y privado a través de la formulación e implementación de proyectos socioeconómicos y planes estratégicos? Sino que también y de manera más amplia y constante en las discusiones, se debería preguntar ¿cómo se han construido sociohistóricamente los problemas que identificamos a nivel empírico?, ¿cuáles son los niveles de realidad en que se ubican los fenómenos de estudio de la planificación?, ¿cuáles procesos sociales, económicos y políticos definen la construcción de las instituciones, de las subjetividades y de las acciones sociales?, ¿tienen los sujetos agencia y capacidad transformadora o están determinados por las estructuras.

En suma, ¿cuál es el papel asignado a la teoría al momento de entender, por ejemplo, la pobreza, la eficiencia institucional, los gustos y preferencias de los sujetos, los mandos medios de las instituciones públicas y el conflicto social?

Por tal motivo, el problema que trata el presente artículo aborda una dimensión empírica y una dimensión teórica, las cuales nacen de la realidad y la experiencia concreta vivencial de aprendizaje del estudiantado al momento de plantear, definir y desarrollar sus diseños de Trabajos Finales de Graduación;), de las percepciones del cuerpo docente sobre ese proceso y sobre su propia práctica docente, en otras palabras, este artículo aborda la realidad de la planificación y sus abordajes desde la perspectiva del diseño de los TFG en el campo de la Planificación. A lo anterior se suman la problematización el perfil de ingreso del estudiantado y las imprecisiones conceptuales que se han identificado en torno a las características técnicas, curriculares y pedagógicas que tienen las MP. Según lo anterior, la pregunta que ha guiado el estudio fue: ¿Cuáles son las características que adquiere la investigación científica dentro de los procesos de Planificación como contribución a la resolución de problemas empíricos? 


\section{Metodología}

Con el objetivo de profundizar en el análisis de la temática propuesta, la dirección de la Maestría en Planificación y Promoción Social ejecutó una investigación cualitativa entre febrero y abril de 2019, para lo cual, primero se realizó una revisión bibliográfica sobre el tema de planificación, las maestrías profesionales y académicas así como las características más comunes de los TFG. Esta revisión se hizo a través de diferentes bases de datos y motores de búsqueda disponibles en la Universidad Nacional (UNA), y en otras universidades públicas ${ }^{3}$. Luego se elaboraron y aplicaron dos cuestionarios en línea dirigidos a las poblaciones más cercanas al desarrollo de las dos maestrías profesionales en Planificación, las personas estudiantes regulares y las personas profesionales egresadas de ambos énfasis del programa. Este primer instrumento contó con la participación de veinticinco personas.

El objetivo principal del cuestionario aplicado al estudiantado fue valorar la claridad que ellos poseen respecto al enfoque teórico, metodología y práctico del trabajo final de graduación, así como la comprensión del componente de investigación diagnóstica y la propuesta de transformación diseñada desde la Planificación.

El segundo instrumento fue aplicado al personal docente de la maestría, por ser consideradas las personas responsables directas del desarrollo del contenido temático correspondiente. Este cuestionario enfatiza en valorar el conocimiento que tienen respecto a las diferencias entre ambos enfoques de las maestrías y los abordajes de los TFG, que permiten valorar la claridad en el proceso de inducción dada al sector estudiantil, a la vez, diseñar una estrategia de fortalecimiento del programa.

Dentro del cuerpo docente entrevistado se contó con la participación del director de la maestría, quien amplió la visión sobre los retos y problemas de la maestría. Se entrevistaron ocho personas del cuerpo docente, además de personas expertas en diseño de programas de maestría y en el Marco Nacional de Cualificaciones de la Educación Superior, con lo cual

3 Entre esas bases de datos se ha consultado: Dialnet, RedALyC, EbscoHost, JSTOR, Researchgate, SciELO entre otras. 
se pretende obtener una percepción desde el área curricular sobre las diferencias entre ambos enfoques de maestrías.

Por su parte, para validar los hallazgos preliminares, se realizó un grupo focal con personas estudiantes que se encuentran iniciando el proceso de preparación de su TFG. Esto con el fin de conocer su percepción y las principales dificultades que están experimentado en ese proceso. Este grupo de discusión fue realizado con cinco estudiantes del énfasis en Planificación estratégica y tres del énfasis de Formulación y gestión de proyectos socioeconómicos, quienes se encontraban finalizando el primer curso de taller de investigación de sus respectivas áreas. En la primera etapa del taller se les solicitó que, de manera libre, expresaran si consideraban que contaban con la suficiente claridad teórica y metodológica para definir y plantear su tema y problema de investigación. Luego, se les expuso los resultados de los instrumentos aplicados al sector estudiantil y docente y se concluyó con un espacio para validar, cuestionar y ampliar los planteamientos expuestos. Todos estos argumentos fueron parte de los insumos que se intentan retomar en el manuscrito.

La presentación de los resultados se realiza de la siguiente manera: En primera instancia se expone el apartado teórico y metodológico de la planificación. Desde esta concepción, se analiza el enfoque de investigación básica aplicada como elementos fundamentales para comprender los enfoques de una maestría profesional y una académica. Luego, se continúa con una breve explicación del enfoque de investigación diagnóstica, muy común en los procesos de planificación. Posteriormente, se desarrolla el apartado relacionado con las características de las maestrías académicas y profesionales, así como la concepción de los TFG en cada una de ellas. Se continua con el apartado de presentación del problema, a partir de la opinión de las personas participantes, un análisis de los hallazgos en el marco de los planteamientos de la planificación ya desarrollados. Finalmente, se presentan las conclusiones y algunas propuestas concretas para el posgrado. 


\section{Perspectiva teórica. La planificación en perspectiva}

La Planificación tiene una larga historia de desarrollo disciplinar que está relacionada con los avances, desarrollos y contradicciones de la creación de los estados nacionales y en las formas de desarrollo económico en el último siglo $\mathrm{XX}$.

En términos generales, se podría decir que la Planificación es el estudio de la gestión de los recursos públicos y privados para la consecución de ciertas metas y objetivos. Es el estudio de cómo las organizaciones sociales utilizan de cierta forma, sus recursos sociales, naturales, financieros, conceptuales, intelectuales para generar valor público o privado. Lo anterior lleva a considerar a la planificación como proceso, compuesto en un primer momento, por su orientación a conocer el problema de interés y un segundo momento a proponer su transformación. Por consiguiente, ya no basta con hacer referencia a las funciones o formas de la planificación (gestión, prospección, coordinación, implementación y evaluación), sino que aparece el desafío de comprender las interacciones entre sus componentes, específicamente, la gestión que de ellas se hace en la práctica (Máttar \& Cuevas, 2017, p.7).

Determinar el objeto de estudio de la Planificación obliga a construir un concepto general y luego a identificar los principales enfoques con los que se ha abordado la planificación en la sociedad. Algunas posiciones teóricas reconocen en la planificación un proceso de análisis político y social de aplicación imperiosa, si realmente se quiere trascender las grandes problemáticas del presente. Otras posiciones, sobre todo de orden administrativo, consideran a la panificación como el uso de diferentes herramientas de apoyo en los procesos de gestión. En ambos casos, la planificación no solo es necesaria, sino indispensable para la gestión de los complejos problemas actuales y futuros.

Negar la planificación es negar la posibilidad de elegir el futuro, es aceptarlo como sea. Si el hombre, un gobierno o una institución renuncian a conducir y se dejan conducir, renuncian a arrastrar y son arrastrados por los hechos, entonces renuncian a la principal libertad humana, que es intentar decidir por nosotros y para nosotros a donde queremos llegar y cómo luchar para alcanzar esos objetivos (Matus, 1987, p.22) 
Por tanto, no sería posible concebir los procesos de planificación sin reflexión crítica de los acontecimientos del pasado y de los fenómenos del presente, sin asumir acciones anticipatorias y propositivas que nos conduzcan un estadio de desarrollo superior, indistintamente del nivel de nuestra intervención. Para Sánchez-Buitrago (2009),

La planificación es un proceso complejo de resignificación en el cual la reflexión como ejercicio comprensivo de una organización, asumida ésta como entidad social viva, histórica, sistémica y autopoiética; como entidad dinámica, es susceptible de lecturas amplias, diversas, plurales a través de las cuales los sujetos que intervienen, a partir de su capacidad de entendimiento y desde su óptica, hacen regresos comprensivos sobre lo sucedido, hacen distanciamientos mentales sobre lo que está pasando y construyen propuestas de anticipación racional de lo venidero, delineando caminos para encaminarse hacia imágenes objetivo del desarrollo que se producen y auto-reproducen en una dinámica ( $p .53)$

En ese sentido, desde una perspectiva académica, la planificación no se enseña como un fin en sí mismo, sino como una mediación entre un presente deficitario y un anhelo o escenario futuro deseado donde la función de planificar está en comprender ese presente desde la consideración socio histórica del fenómeno para construir a partir de esto el futuro deseado y posible y, finalmente, diseñar la estrategia más adecuada para lograrlo. Así, la planificación es una construcción humana que se realiza en el marco de contextos complejos, en donde las relaciones sociales, económicos, políticos, ambientales, culturales, religiosas no son ni aisladas ni inconexas.

En realidad, el futuro se construye mediante un delicado equilibrio sobre un camino de doble vía entre las realidades presentes y los escenarios posibles y deseables. El arte de transitar intelectual y políticamente en ambos sentidos es precisamente el nombre del juego y éste no es otro que el de definir e instrumentar una estrategia de desarrollo (Martín, 2005, p.11).

A esa estrategia de desarrollo, es a la que refiere la propuesta de planificación, previa reflexión y análisis crítico de la problemática de interés.

Desde una perspectiva más concreta y aplicada, la planificación también puede considerarse como la definición de procedimientos para influir en 
la lógica natural de los acontecimientos. En esa línea Ander-Egg (1991) indica que la planificación es;

La acción consistente en utilizar un conjunto de procedimientos mediante los cuales se introduce una mayor racionalidad y organización en un conjunto de actividades y acciones articuladas entre sí que, previstas anticipadamente, tienen el propósito de influir en el curso de determinados acontecimientos, con el fin de alcanzar una situación elegida como deseable, mediante el uso eficiente de medios y recursos (p.25).

En términos específicos, se podrían identificar cuatro grandes escuelas de planificación (Lira, 2006, pp. 10-42) que presentan diferentes enfoques o abordajes, así como diferentes objetos de planificación: la Planificación Tradicional; la Planificación Situacional; la Planificación Estratégica y la Planificación Prospectiva. Estas escuelas tienen importantes diferencias epistemológicas y metodológicas, así como diferentes contextos sociopolíticos de los que provienen. Al mismo tiempo coinciden en que la planificación puede tener una dimensión estratégica normativa, operativa, y ser también analizada como un objeto socio histórico y político de la organización social como un todo.

En la Escuela de Planificación y Promoción Social (PPS), la planificación se concibe como un proceso de investigación y análisis sistemático del pasado y del presente, pero con una perspectiva de futuro que permite enfocar la acción hacia un horizonte superior. Desde la docencia se promueven y enseñan los diferentes niveles de abordaje en los procesos de planificación, pero en la práctica, los proyectos hacia afuera se han concentrado principalmente en el enfoque meso y micro social, lo que deja al pendiente abordar problemas desde un enfoque macro social. Se podría afirmar que el origen tanto de la Universidad Nacional de Costa Rica como de la Escuela de Planificación y Promoción Social, marcaron el foco de acción de sus programas de investigación y extensión hacia la organización y hacia espacios locales, en los cuales se ha reconocido, validado y construido un potencial teórico y metodológico que guía los procesos de análisis de los problemas de la organización desde la planificación. Pero también se ha reconocido y consolidado el papel estratégico de la participación de los actores locales como gestores de su propio proceso de cambio al desarrollar desde la Unidad Académica, la metodología de planificación participativa. 
Respecto a la Maestría de Planificación, tiene una orientación profesional, se ha abocado a los niveles meso y micro sociales de la planificación. Uno de los objetivos establecidos en su perfil de salida es que el estudiantado cuente con herramientas para el análisis de una realidad en particular y tenga la capacidad de formular propuestas de mejora de esa realidad a través de distintos métodos de planificación.

El problema radica en entender precisamente este último punto. Con base en ello, cabe preguntarse: ¿Cómo se entiende la realidad concreta y desde cuáles presupuestos teóricos y metodológicos se puede partir para presentar una solución viable y factible?, ¿cuál es la lógica intelectual y programática general que se debe seguir para lograr unir esas dos dimensiones de la planificación?, ¿cómo se expresa finalmente en el formato de un TFG?

Tradicionalmente, el ciclo del proyecto, como se conoce en planificación otorga luces importantes para dar respuesta a las anteriores preguntas. Se identifica y se analiza preliminarmente un problema de la realidad en el ámbito de la planificación, se determinan sus causas y efectos, pero a la vez, se hace un análisis orientado a conocer las relaciones e interacciones entre los componentes de esa realidad y, como lo plantea Mattar (2017), para comprender la gestión que de ellas se hace en la práctica. Desde ahí se derivan posibles soluciones que tienen criterios de viabilidad o de factibilidad, según corresponda. Sin embargo, uno de los problemas identificados en los últimos años es que esta idea no es totalmente clara entre personal docente y estudiantes, la cual se expresa con mayor claridad en la desconexión que existe entre el proceso de investigación y la formulación de la propuesta de solución. Es decir ¿cuál es la conexión existente entre los presupuestos conceptuales que han identificado un problema como tal y que han analizado sus dimensiones causales con las propuestas de solución?, ¿brindan los cursos de la Maestría en Planificación las habilidades para lograr esta conexión?

Algunas personas estudiantes han expresado que los métodos, los instrumentos y las herramientas de planificación han sido insuficientes para este propósito. Por otro lado, otras personas estudiantes han indicado tener pocos fundamentos teóricos para analizar y explicar la realidad y que esta situación está ligada con el perfil de entrada multidisciplinario que existe. Otro sector estudiantil ha identificado problemas de interconexión entre 
los cursos de investigación y construcción de objetos de estudio con los cursos prácticos o los más instrumentales.

En resumen, el problema estudiado es acerca de la conexión lógica entre las dos etapas de la planificación: la etapa de investigación (conocer la situación problemática) y la etapa de planificación (diseñar la estrategia de transformación, sea desde la planificación estratégica o desde la gestión de proyectos socioeconómicos) y, para ello es necesario desarrollar cursos teóricos, metodológicos y prácticos. En este sentido, el reto radica en cómo fortalecer el perfil de salida profesional a través de la reorientación de las bases investigativas para la identificación y el análisis de realidades concretas en el campo de la planificación.

Terminamos este apartado citando a Sánchez (2009) respecto a su concepción sobre planificación, la cual nos invita a innovar: "La planeación es una esperanza creadora en un mundo que se mueve en una desesperanza colectiva, por eso está claro que el reto que debemos enfrentar, académica y socialmente, ahora y mañana, es su resignificación" (p.57).

\section{La investigación básica aplicada desde la planificación}

Cervo y Bervian (1989) indican que "se define la investigación como una actividad encaminada a la solución de problemas. Su objetivo consiste en hallar respuestas a preguntas mediante el empleo de procesos científicos" (p. 41). Como se indican los actores, la investigación normalmente se ha visto reducida a una actividad sistemática ordenada que pretende solucionar un problema, sin embargo, muchas veces se deja de lado que fundamentalmente la ciencia es el acto reflexivo de construir problemas, y no cualquier problema, sino problemas científicos

Según Torres Carrillo y Jiménez Becerra (2004):

Plantear un problema de investigación equivale a delimitar dentro de un contexto teórico, disciplinar, tecnológico o profesional un objeto de indagación, un interrogante o un conjunto de interrogantes cuyo abordaje generará nuevos conocimientos o validará los existentes en nuevos contextos (p. 16). 
El problema de investigación es una construcción intelectual, que se deriva de la reflexión de los componentes empíricos de la realidad y de los marcos interpretativos con que la persona que investiga, entiende, analiza y explica la realidad. De esta manera, cabe plantear ¿Cuál es el proceder para la construcción de un problema científico? Y con base en esa reflexión ¿cuáles son los típicos problemas que la planificación busca solucionar?

Cerdas (1991) identifica algunos tipos de problemas: problemas empíricos, problemas conceptuales y problemas generales, específicos y particulares. Cada uno de ellos se ubica, como construcción epistemológica, en diferentes niveles de a realidad. Sin embargo, independientemente de si el problema es conceptual o empírico ubicado en un contexto organizacional- institucional el problema sigue siendo de carácter teórico-interpretativo, en donde los atributos de la ciencia como coherencia, rigurosidad, parsimonia, fiabilidad, verificabilidad deben estar presentes durante todo el proceso de investigación (pp. 143-147). Como plantean Torres Carrillo y Jiménez Becerra 2004:

[L]a formulación de problemas de investigación no es un asunto técnico, sino un desafío teórico y epistemológico que implica un esfuerzo del pensamiento que debe reconocer los límites de "lo ya sabido" y atreverse a preguntar por "lo inédito", lo no pensado hasta el momento, pero susceptible de ser pensado e investigado desde el acumulado del campo de conocimiento en el que se localiza dicho problema (p.17).

Se habla de una investigación pura o básica cuando se produce un nuevo conocimiento o se pretende incrementar los postulados teóricos de una ciencia determinada. Se dice que una investigación es aplicada cuando el conocimiento busca tener una aplicación inmediata en la solución de problemas prácticos (Arias, 2012, p.22).

Asimismo, Arias (2012) coincide con MCESCA (Marco de Cualificaciones para la Educación Superior Centroamericana) al ubicar la investigación cuya motivación es la solución de problemas prácticos a la investigación aplicada y la investigación cuyo propósito es el aporte al desarrollo conceptual, a la investigación básica. 
Para Lozada (2014):

La generación de una teoría científica a partir de una idea o de la constatación de un fenómeno físico se denomina investigación fundamental o básica. Mientras que la investigación aplicada tiene por objetivo la generación de conocimiento con aplicación directa y a mediano plazo en la sociedad o en el sector productivo (p.35)

Una MA, se encarga de utilizar la investigación básica para abordar sus objetos de estudio, de tal forma que se produzcan nuevos conocimientos. Mientras que las MP, utilizan investigación aplicada, en la cual se pretende generar resultados con aplicación directa.

Hernández, Saavedra y Sanabria (2006) consideran que el objeto de estudio es:

La delimitación de un campo específico del mundo de la vida y del trabajo (...) que se decide privilegiar y alrededor del cual se emprende una labor de índole teórica (construcción de conceptos y de relaciones lógicas entre ellos) y metodológica (definición de modos apropiados de intervenir en la realidad a la luz de dichos conceptos (p.25).

Para Brenes Chacón (1987) un trabajo final de graduación, "constituye la última actividad académica que debe realizar una estudiante para hacerse merecedor de determinado título universitario". El autor señala que tiene entre sus propósitos fundamentales:

a) Utilizar los conocimientos y experiencias producto del proceso de formación para la identificación, análisis y proposición de soluciones a problemas relevantes y concretos

b) Emplear métodos y técnicas de estudio o investigación propios de la disciplina

c) Demostrar capacidad y madurez intelectual, al igual que creatividad científica y profesional dentro del campo estudiado (p.27). 
Como se puede apreciar estas preguntas si bien buscan una solución práctica, tendrán su profundidad investigativa dependiendo de que tanto el investigador desee explicar los fenómenos que llevaron a la creación de las problemáticas de las preguntas anteriores y que tan efectiva se espera sea la solución.

\section{Principales características de las maestrías profesionales y las maestrías académicas}

El Consejo Nacional de Rectores de Costa Rica (CONARE), diferencia una MA de una MP en cuanto a la finalidad del conocimiento y las características curriculares en cada caso:

La maestría académica profundiza y actualiza conocimientos principalmente para realizar investigación que genere más conocimiento, por lo que ésta se constituye en su núcleo generador. Su plan de estudios es más individualizado por estudiante Co(los cursos) estarán dedicados a actividades de investigación. [Mientras que] la maestría profesional profundiza y actualiza conocimiento, con el objeto primordial de analizarlo, sintetizado, transmitido y solucionar problemas (CONARE, 2012, pp.109-110).

Desde esta definición se puede identificar una diferencia entre ambas modalidades: por un lado, la maestría académica se enfoca en analizar la realidad buscando respuestas científicas a los fenómenos, procesos, decisiones y accionares que se producen en la dinámica de las diferentes estructuras que conforman la sociedad. La maestría profesional, por su parte, "profundiza y actualiza conocimiento, con el objeto primordial de analizarlo, sintetizarlo, transmitirlo y solucionar problemas" (Conare, 2012). Con esta conceptualización lo que se indica es que las MP pretenden la implementación de alternativas y propuestas a los problemas presentes en la realidad, lo cual incluye la etapa de investigación de dicha problemática. En el caso de las MA, construyen conocimientos a partir de investigaciones previas.

El Marco de Cualificaciones para la Educación Superior Centroamericana (MCESCA, 2018) presenta los resultados de aprendizaje esperados para los siguientes peldaños educativos, basados en la Clasificación Internacional Normalizada de la Educación (CINE): 
1. Técnico superior universitario. Nivel CINE 5 - Educación terciaria de ciclo corto

2. Bachillerato universitario. Nivel CINE 6 - Educación terciaria de ciclo corto

3. Licenciatura Nivel CINE 6 - Educación terciaria o nivel equivalente

4. Maestría. CINE 7 - Nivel de maestría, especialización o equivalente

5. Doctorado CINE 8- Nivel de doctorado o equivalente

En el caso de la CINE 7, es decir, nivel de maestría, hace una importante y pertinente distinción de acuerdo con los resultados de aprendizaje esperados ya que permite tener mayor claridad sobre los diferentes componentes pedagógicos y los perfiles de salida en cada caso como se puede apreciar en la Tabla 1. 


\section{Tabla 1}

Resultados de aprendizaje de las MA y MP

\begin{tabular}{|c|c|c|}
\hline Maestría & Maestría Profesional & Maestría Académica \\
\hline Descriptores & \multicolumn{2}{|c|}{ Resultados de aprendizaje esperados } \\
\hline \multirow[t]{4}{*}{$\begin{array}{l}\text { Saberes dis- } \\
\text { ciplinarios y } \\
\text { Profesionales }\end{array}$} & $\begin{array}{l}\text { Demuestra conocimiento am- } \\
\text { plio de la teoría y práctica de un } \\
\text { campo profesional especializado } \\
\text { en contextos multidisciplinarios. }\end{array}$ & $\begin{array}{l}\text { Demuestra conocimiento } \\
\text { profundo de la teoría y prác- } \\
\text { tica de un campo disciplinar } \\
\text { especializado en contextos } \\
\text { multidisciplinarios. }\end{array}$ \\
\hline & $\begin{array}{l}\text { Demuestra conocimiento de } \\
\text { cultura humanística, de marcos } \\
\text { axiológicos, de sustentabilidad } \\
\text { en los campos de su profesión. }\end{array}$ & $\begin{array}{l}\text { Demuestra conocimiento de } \\
\text { cultura humanística, de mar- } \\
\text { cos axiológicos, de susten- } \\
\text { tabilidad en los campos de } \\
\text { investigación. }\end{array}$ \\
\hline & $\begin{array}{l}\text { Muestra conocimiento sobre el } \\
\text { riesgo de desastres en el campo } \\
\text { de su especialidad y propone so- } \\
\text { luciones para reducirlo. }\end{array}$ & $\begin{array}{l}\text { Muestra conocimiento sobre } \\
\text { el riesgo de desastres en el } \\
\text { campo de su especialidad } \\
\text { y propone soluciones para } \\
\text { reducirlo. }\end{array}$ \\
\hline & $\begin{array}{l}\text { Realiza innovaciones tecnológi- } \\
\text { cas en su campo profesional. }\end{array}$ & $\begin{array}{l}\text { Realiza innovaciones me- } \\
\text { todológicas en su campo } \\
\text { profesional. }\end{array}$ \\
\hline
\end{tabular}




\begin{tabular}{lll}
\hline Maestría & Maestría Profesional \\
\hline Aplicación & Demuestra pensamiento crítico, \\
de conoci- & actitud investigativa y rigor ana- \\
mientos, re- & lítico en el planteamiento y la re- \\
solución de & solución de problemas comple- \\
problemas e jos y emergentes. \\
innovación
\end{tabular}

Identifica y resuelve problemas complejos en entornos nuevos o emergentes, de manera innovadora, dentro de contextos multi e interdisciplinarios.

Propone e implementa nuevos procedimientos y metodologías aplicables a la solución de problemas complejos que requieren abordajes multi $\mathrm{e}$ interdisciplinarios.

Toma decisiones profesionales y propone escenarios alternativos de solución utilizando métodos $y$ estrategias especializadas de análisis, manejo y generación de información y evalúa su eficacia, implicaciones y consecuencias.

\section{8}

Demuestra habilidad en la selección, uso y adaptación de herramientas metodológicas, tecnológicas, equipo especializado y de alta tecnología, y en la lectura e interpretación de datos pertinentes a la solución de problemáticas complejas específicas.

\section{Maestría Académica}

Demuestra pensamiento crítico, actitud investigativa y rigor analítico y metodológico en el planteamiento y la resolución de problemas complejos y emergentes.

Identifica y resuelve problemas teórico-metodológicos complejos o emergentes, de manera innovadora, dentro de contextos multi e interdisciplinarios.

Propone e implementa nuevos enfoques teóricos y metodológicos aplicables a la solución de problemas complejos que requieren abordajes multi e interdisciplinarios.

Toma decisiones sobre problemas teóricos y metodológicos, propone escenarios alternativos de solución, utilizando métodos de investigación y estrategias especializadas de análisis, manejo y generación de información y evalúa su eficacia, implicaciones y consecuencias.
Demuestra habilidad en el uso de herramientas metodológicas y de alta tecnología para experimentación y procesamiento e interpretación de datos.

Daniel Láscarez Smith, Ángel Abelino Ortega, Luis Fernando Morales-Abarca

\section{(ㄷ)(1)(2) (2)}




\begin{tabular}{|c|c|c|}
\hline Maestría & Maestría Profesional & Maestría Académica \\
\hline \multirow[t]{4}{*}{$\begin{array}{l}\text { A u t o n om ía } \\
\text { con respon- } \\
\text { sabilidad per- } \\
\text { sonal, laboral } \\
\text { y social }\end{array}$} & $\begin{array}{l}\text { Demuestra responsabilidad y } \\
\text { autonomía profesional para el } \\
\text { desempeño de sus actividades } \\
\text { profesionales, dentro de los mar- } \\
\text { cos normativos y éticos de su } \\
\text { campo profesional. }\end{array}$ & $\begin{array}{l}\text { Demuestra responsabilidad } \\
\text { y autonomía profesional aca- } \\
\text { démica para el desempeño } \\
\text { de sus actividades académi- } \\
\text { cas profesionales, dentro de } \\
\text { los marcos normativos y éti- } \\
\text { cos de su disciplina. }\end{array}$ \\
\hline & $\begin{array}{l}\text { Analiza críticamente las princi- } \\
\text { pales perspectivas y tendencias } \\
\text { nacionales e internacionales en } \\
\text { su campo profesional para la } \\
\text { mejora continua de su práctica. }\end{array}$ & $\begin{array}{l}\text { Analiza críticamente las prin- } \\
\text { cipales perspectivas y ten- } \\
\text { dencias nacionales e inter- } \\
\text { nacionales en su campo de } \\
\text { estudio para la mejora conti- } \\
\text { nua de su trabajo académico. }\end{array}$ \\
\hline & $\begin{array}{l}\text { Evalúa su desempeño profesio- } \\
\text { nal con base en referentes de } \\
\text { calidad, nacionales e internacio- } \\
\text { nales, y la incidencia de sus de- } \\
\text { cisiones en los aspectos huma- } \\
\text { nos, sociales, ambientales y de } \\
\text { reducción de riesgos. }\end{array}$ & $\begin{array}{l}\text { Evalúa su desempeño aca- } \\
\text { démico profesional con base } \\
\text { en referentes de calidad, na- } \\
\text { cionales e internacionales, y } \\
\text { la incidencia de sus decisio- } \\
\text { nes en los aspectos huma- } \\
\text { nos, sociales, ambientales y } \\
\text { de reducción de riesgos. }\end{array}$ \\
\hline & $\begin{array}{l}\text { Identifica y emprende proyectos } \\
\text { profesionales para la generación } \\
\text { de negocios y para el benefi- } \\
\text { cio social con criterios de per- } \\
\text { tinencia, calidad, innovación y } \\
\text { sustentabilidad. }\end{array}$ & $\begin{array}{l}\text { Identifica y emprende pro- } \\
\text { yectos académicos profe- } \\
\text { sionales para la generación } \\
\text { de patentes y aplicaciones } \\
\text { con criterios de pertinen- } \\
\text { cia, calidad, innovación y } \\
\text { sustentabilidad. }\end{array}$ \\
\hline
\end{tabular}




\begin{tabular}{|c|c|c|}
\hline Maestría & Maestría Profesional & Maestría Académica \\
\hline \multirow[t]{3}{*}{ Comunicación } & $\begin{array}{l}\text { Comunica a público especiali- } \\
\text { zado o no, información de su } \\
\text { campo profesional en varios } \\
\text { lenguajes y formatos de manera } \\
\text { asertiva, clara, rigurosa y preci- } \\
\text { sa, con el uso apropiado de re- } \\
\text { cursos tecnológicos. }\end{array}$ & $\begin{array}{l}\text { Comunica a público especia- } \\
\text { lizado o no, información de } \\
\text { su campo académica profe- } \\
\text { sional en varios lenguajes y } \\
\text { formatos y en los medios es- } \\
\text { pecializados del ámbito pro- } \\
\text { fesional disciplinar de mane- } \\
\text { ra asertiva, clara, rigurosa y } \\
\text { precisa, con el uso apropiado } \\
\text { de recursos tecnológicos. }\end{array}$ \\
\hline & $\begin{array}{l}\text { Se comunica correctamente en } \\
\text { su lengua oficial y utiliza una } \\
\text { lengua extranjera con el dominio } \\
\text { requerido para el ejercicio de su } \\
\text { profesión, con uso apropiado de } \\
\text { vocabulario y cumpliendo con } \\
\text { las normas de comunicación } \\
\text { propias de la profesión y están- } \\
\text { dares internacionales. }\end{array}$ & $\begin{array}{l}\text { Se comunica correctamente } \\
\text { en su lengua oficial y utiliza } \\
\text { una lengua extranjera con } \\
\text { el dominio requerido para el } \\
\text { ejercicio de su profesión, con } \\
\text { uso apropiado de vocabulario } \\
\text { y cumpliendo con las normas } \\
\text { de comunicación propias de } \\
\text { la profesión y estándares } \\
\text { internacionales. }\end{array}$ \\
\hline & $\begin{array}{l}\text { Utiliza tecnologías digitales para } \\
\text { modelamiento, manejo e inter- } \\
\text { pretación de datos e información } \\
\text { de forma apropiada a su nivel y } \\
\text { su profesión. }\end{array}$ & $\begin{array}{l}\text { Utiliza tecnologías digitales } \\
\text { para modelamiento, manejo } \\
\text { e interpretación de datos e in- } \\
\text { formación de forma apropia- } \\
\text { da a su nivel y su profesión. }\end{array}$ \\
\hline
\end{tabular}




\begin{tabular}{|c|c|c|}
\hline Maestría & Maestría Profesional & Maestría Académica \\
\hline \multirow[t]{5}{*}{$\begin{array}{l}\text { Interacción } \\
\text { profesional, } \\
\text { cultural y } \\
\text { social }\end{array}$} & $\begin{array}{l}\text { Demuestra habilidades colabo- } \\
\text { rativas y cooperativas en el cam- } \\
\text { po profesional, cultural y social. }\end{array}$ & $\begin{array}{l}\text { Demuestra habilidades cola- } \\
\text { borativas y cooperativas en } \\
\text { el campo profesional, cultural } \\
\text { y social. }\end{array}$ \\
\hline & $\begin{array}{l}\text { Integra y lidera con visión estra- } \\
\text { tégica equipos de trabajo para } \\
\text { desarrollar el potencial del gru- } \\
\text { po y estimular la productividad } \\
\text { profesional. }\end{array}$ & $\begin{array}{l}\text { Integra y lidera con visión es- } \\
\text { tratégica equipos de trabajo } \\
\text { para desarrollar el potencial } \\
\text { del grupo y estimular la pro- } \\
\text { ductividad académica. }\end{array}$ \\
\hline & $\begin{array}{l}\text { Muestra respeto hacia la diversi- } \\
\text { dad en todas sus manifestacio- } \\
\text { nes y contribuye al bien común. }\end{array}$ & $\begin{array}{l}\text { Muestra respeto hacia la di- } \\
\text { versidad en todas sus mani- } \\
\text { festaciones y contribuye al } \\
\text { bien común. }\end{array}$ \\
\hline & $\begin{array}{l}\text { Establece alianzas estratégicas } \\
\text { con socios nacionales, extranje- } \\
\text { ros y redes de colaboración que } \\
\text { fortalecen el trabajo profesional } \\
\text { y proyectos conjuntos. }\end{array}$ & $\begin{array}{l}\text { Establece alianzas estraté- } \\
\text { gicas con socios naciona- } \\
\text { les, extranjeros, y redes de } \\
\text { colaboración que fortalecen } \\
\text { la investigación, publicación } \\
\text { conjunta y demás procesos } \\
\text { académicos. }\end{array}$ \\
\hline & $\begin{array}{l}\text { Participa y promueve en contex- } \\
\text { tos académicos y profesionales } \\
\text { la innovación, la transferencia } \\
\text { del conocimiento y el avance } \\
\text { científico tecnológico, social y } \\
\text { cultural. }\end{array}$ & $\begin{array}{l}\text { Participa y promueve en } \\
\text { contextos académicos y } \\
\text { profesionales la innovación, } \\
\text { la transferencia del conoci- } \\
\text { miento y el avance científico } \\
\text { tecnológico, social y cultural. }\end{array}$ \\
\hline
\end{tabular}

Fuente: MCESCA, 2018.

De la tabla se deduce una serie de similitudes en ambos tipos de maestrías, dentro de las cuales se resalta el énfasis que se le da al conocimiento de la teoría y de la práctica como punto de partida en los procesos de estudio, así como la importancia a los procesos de investigación y al uso de diferentes tipos de instrumentos de investigación. Sin embargo, se debe indicar que mientras la maestría profesional pretende comprender 
el fenómeno para proponer nuevos procedimientos y métodos para la solución de los problemas de interés, la maestría académica se enfoca a comprender el fenómeno estudiado para solucionar problemas teóricos o metodológicos, esto a partir de propuestas de nuevas construcciones y miradas teóricas o metodológicas, caracterizas por la interdisciplinariedad.

De acuerdo con el planeamiento de MCESCA (2018) ambas maestrías se caracterizan por realizar un análisis crítico de las perspectivas y tendencias nacionales de sus respectivos campos profesionales. También, se observan importantes diferencias entre los dos tipos de maestrías, las cuales se enfocan primordialmente en su objeto de estudio. La maestría profesional pretende diseñar estrategias de transformación, mientras que la maestría académica pretende aportar a la teoría o a las metodologías correspondientes a sus áreas de estudio. Estos elementos serán abordados en las siguientes páginas, a partir del análisis del caso de la Maestría en planificación.

\section{El diagnóstico como método de investigación en la planificación.}

Ander-Egg y Aguilar Idañez (2013) señalan que "el término "diagnóstico" proviene del griego diagnoslikós, formado por el prefijo día. "a través", y gnosis, "conocimiento", "apto para El término "diagnóstico" proviene del griego diagnoslikós, formado por el prefijo día. "a través", y gnosis, "conocimiento", "apto para" (pp. 12-13).

La investigación diagnóstica es un tipo de investigación aplicada que busca generar conocimiento para la inmediata intervención social. Tal como lo mencionan Nato y Carbajal (2011), "la investigación diagnóstica, como momento privilegiado de articulación entre conocimiento e intervención, se orienta a detectar, describir, y/ o comprender las complejas relaciones entre sujetos (actores), prácticas (acciones) y escenarios (estructura)" (p. 10).

Es una herramienta para la acción de gran potencial heurístico que permite sentar bases científicas sobre el análisis de una situación específica. Al ser aplicado, como se dijo anteriormente, sirve como herramienta de acción y por tanto se ubica dentro de un proceso de intervención social.

Respecto al concepto de heurística, Delgado (2010) señala que es una "palabra que proviene del término griego heurisko que quiere decir yo 
busco, descubro, y que es el método que se usa para encontrar lo nuevo, lo que se desconoce".

En la planificación la utilización del diagnóstico como herramienta de investigación está muy difundida y con ella, se busca tener una línea base o conocimiento previo de los fenómenos o contextos que determinan y definen al objeto de estudio. A su vez, con base en estos criterios se busca generar un proceso de intervención que permita la solución de problemáticas presentes en la realidad, o cuando menos que contribuya en la solución que corresponda.

Ya sea una organización que requiere un plan estratégico para su fortalecimiento organizacional o para el aprovechamiento óptimo de sus recursos, un análisis estratégico de su desenvolvimiento, un alineamiento estratégico de algunos de sus componentes o una organización (pública o privada) que aprovecha una idea $u$ oportunidad para desarrollar un proyecto con enfoque social o financiero. Estas u otras estrategias diseñadas desde la planificación, requieren del diagnóstico como proceso investigativo previo a su diseño. Es por ello que, en los diseños para los TFG de la maestría de planificación, esta etapa deberá ser robusta dado que le permitirá al estudiante tener un mayor conocimiento de la dinámica que rodea al objeto de estudio. De esta manera, es posible proponer los mecanismos o alternativas que permitan transformar esa realidad en una más conveniente para los actores afectados.

En el caso de las maestrías que se presentan como profesionales, como las de la Escuela de Planificación, la labor diagnóstica no debe verse como un elemento técnico, sino como un proceso analítico a la hora de buscar alternativas de solución a los problemas de interés para los profesionales. La investigación diagnóstica no es una forma más "fácil" de hacer investigación, pues el componente reflexivo para la construcción del problema científico sigue estando presente, aunque sea una organización, una empresa o una institución, el campo de la aparición fenomenológica de sus efectos, conflictos o consecuencias. 


\section{Percepción estudiantil y del cuerpo docente acerca del papel de la investigación en la formulación de propuestas de graduación en el campo de la planificación}

Como parte de la investigación se entrevistó a ocho personas docentes de la Maestría de Planificación en los énfasis de Planificación Estratégica y Formulación, y Gestión de Proyectos Socioeconómicos. A este grupo de personas se les preguntó sobre varios elementos relacionados con el perfil de salida de las personas egresadas en ámbitos profesionales y académicos, las dificultades abordadas en estos procesos y el papel que tiene la investigación para fortalecer el perfil de salida establecido. También, se entrevistó a veinticinco personas estudiantes de la maestría en Planificación sobre los mismos aspectos, más otras valoraciones relacionadas con el proceso de diseño de sus trabajos de graduación, sobre sus expectativas y concepciones sobre la investigación, así como sobre su percepción respecto a los cursos de investigación y el aporte que estos tienen para formular la propuesta de TFG.

Uno de los hallazgos más relevantes es que las personas docentes no identifican claramente las diferencias metodológicas y epistemológicas entre una MP y una MA, por ejemplo, identifican aspectos de forma como que el TFG es diferente, pues en la maestría académica se hace una tesis y en la profesional se hace un proyecto. Es decir, identifican las diferencias en función de la forma del trabajo no en sus aspectos teóricos y metodológicos.

También indicaron que la MP tiene un enfoque hacia "la vida profesional"; y la maestría académica, para la "vida académica". Esta idea en realidad no evoca una diferencia ni apela la realidad del trabajo científico básico o en la ciencia aplicada. Por ese motivo, se debería abordar la idea de que los problemas institucionales deberían ser estudiados con bases teóricas, metodológicas, sistemáticas y claras. Otro punto que se identificó fue la discrepancia de las personas docentes en cuanto al perfil de ingreso y el perfil de salida del sector estudiantil de la maestría. Para algunas personas docentes no es relevante ese perfil de entrada, solo el de salida, pero tampoco tienen claro cómo debería ser la estrategia de mediación para nivelar a estudiantes que provienen de otras profesiones totalmente diferentes a la planificación, por ejemplo, Veterinaria, alguna Ingeniería, o bien, estudiantes de Ciencias Sociales. Tampoco hay claridad de que las estrategias utilizadas para diseñar el proceso pedagógico deberían tomar

Daniel Láscarez Smith, Ángel Abelino Ortega, Luis Fernando Morales-Abarca

\section{(C) (1)(2)}


en consideración los diferentes niveles de conocimiento sobre lo social, institucional y la investigación en ciencias sociales.

De los aportes emitidos por el sector docente se concluye que no existe uniformidad de criterios ni la claridad necesaria sobre los abordajes teóricos y metodológicos para diferenciar los TFG de una maestría profesional y una académica. Aunque en un sector de las personas docentes participantes, existe coincidencia con los planteamientos de MCESCA al concebir que, en ambos tipos de maestrías, se requiere pensamiento crítico y mucho rigor analítico en los planteamientos de las propuestas elaboradas desde los trabajos finales. En la maestría profesional, según algunas personas docentes, se pretende proponer nuevos procedimientos metodológicos para la solución de problemas asociados a las organizaciones o las empresas; mientras que, en la maestría académica, el énfasis está, en hacer propuestas de nuevos enfoques teóricos y metodológicos orientados a la solución de problemas. Es clara la necesidad de discutir y unificar algunos criterios en el personal docente, con el objeto de mejorar el abordaje de todos aquellos cursos de la malla curricular que están de alguna manera asociados a la definición, planteamiento o desarrollo de los trabajos finales de las maestrías.

Por su parte, el objetivo de las entrevistas con las personas estudiantes fue identificar las valoraciones sobre las concepciones de práctica académica y profesional sobre las dificultades entorno a la formulación del problema de investigación y el uso de teorías, métodos y técnicas de investigación. Uno de los hallazgos es que los estudiantes consideran que la base para el buen desempeño en las maestrías profesionales es el dominio de técnicas e instrumentos de planificación; mientras que la base para el desempeño en las maestrías académicas es el uso correcto del método científico. De esto se desprende la separación entre ciencia y técnica (académica y profesional).

Otro importante hallazgo es que el papel asignado a la investigación por parte del personal decente está determinado por la profundidad, el alcance y la aplicabilidad de la investigación, pero la concepción de aplicabilidad está directamente relacionada con praxis. En otras palabras, la concepción de investigación está orientada hacia la investigación aplicada, pero no es el producto final ni el más importante. El estudiantado, por su parte, asignó un rol diferenciado con respecto a la profundidad y la utilidad del conocimiento generado en cada proceso educativo. Es decir, no hubo 
claridad en señalar la importancia de la investigación con respecto a su proceso personal de investigación.

Cuando se les consultó sobre el tipo de investigación que está realizando el estudiantado para elaborar los TFG, y si la maestría podría promover investigaciones básicas y aplicadas, hubo diferentes posiciones. Algunas personas indicaron que una maestría profesional debe enfocarse en los trabajos de proyectos que tienen orientación en la solución de problemas o promoción de la innovación, lo cual sugiere que las investigaciones deben ser aplicadas y no básicas o de alto nivel de abstracción. No obstante, pareciera que el papel de la investigación surge después de haber presentado el plan estratégico o el proyecto y no al revés: la investigación como base para la construcción de la propuesta de proyectos.

Otras personas profesionales respondieron que la estructura curricular no permite enfocarse en promover investigaciones básicas y que el perfil docente tampoco está definido para esos fines. Un tercer grupo piensa que si se pueden o deberían ejecutar investigaciones básicas.

Se evidencia que la respuesta a las preguntas orientadas a conocer la claridad que tiene el sector estudiantil, respecto al enfoque metodológico y al abordaje epistemológico de la investigación, son muy diferentes de acuerdo con la formación básica de las personas estudiantes. Cuando la formación es en el campo de las Ciencias sociales, existe mayor claridad al respecto, no así cuando la formación ha sido realizada en otras áreas del saber. Esto induce a la unidad académica a reconsiderar el perfil de entrada de las futuras personas que ingresen a la maestría o a repensar algunos cursos de nivelación que permitan homogeneizar conceptos disciplinares.

Algunas personas entrevistadas consideran que la investigación de la maestría profesional se debería enfocar principalmente a hacer propuestas reales y acordes a una situación conocida por la organización y que la etapa de investigación no debería de tener mayor profundidad, por cuanto las evidencias empíricas ya definen el problema.

Sobre la formación que el programa les brinda en el tema de investigación, existe una percepción crítica de parte del sector estudiantil, sobre todo porque considera que la falta de criterios con algún grado de homogeneidad entre el personal docente a cargo de cursos relacionados genera 
confusión y retrasa su salida del programa. Por ejemplo, una respuesta emitida sostiene: "Los profesores tanto de taller como de Investigación tenían enfoques distintos, no se ponían de acuerdo y eso creó mucho tiempo perdido, confusión y frustración".

El grupo focal permitió cerrar el proceso con una discusión crítica. Como elemento positivo, se rescata la valoración que se hace respecto a la necesidad de fortalecer la función de planificación en las empresas, las organizaciones y los espacios locales, para lo cual, el programa de la maestría en Planificación resulta muy pertinente. Desde una perspectiva crítica, se plantea que la maestría está diseñada como un programa más académico mientras que el interés de la mayoría de las personas estudiantes es de un programa más instrumental y herramental que les permita desde la Planificación, aportar a la solución de problemas conocidos en sus espacios laborales.

Se reconoce la necesidad de profundizar el análisis de las problemáticas, pero el énfasis de los trabajos finales debiera de estar, según el criterio de muchas personas, en la propuesta de planificación, por lo que se debe fortalecer la formación disciplinar. Uno de los planteamientos al respecto sostiene lo siguiente: "La historia es importante, pero se necesitan más herramientas de investigación y aplicación en los campos de trabajo".

De los diferentes aportes empíricos generados a través de los instrumentos aplicados, se determina la falta de claridad y de uniformidad de criterios en el equipo docente, respecto al enfoque teórico y metodológicos de la investigación o el TFG en la maestría. Lo anterior representa una debilidad que se transmite al sector estudiantil y, que, de alguna manera, genera mayor confusión y dificultad sobre todo en la etapa de planteamiento y justificación del tema y el problema de investigación. Esto ha llevado, en algunos casos, a considerar que un trabajo final de las maestrías profesionales consiste en una propuesta de planificación que surge a partir de una percepción de parte de la organización sobre un problema particular, lo que obvia o desmerita la etapa de investigación, o cuando menos, su nivel de profundidad.

Como resultado de esta investigación, la Escuela de Planificación y Promoción Social ha iniciado una etapa de análisis del programa de posgrados con el objeto de hacer replanteamientos que permita fortalecer la oferta en beneficio del sector estudiantil y de las organizaciones o espacios 
laborales de las personas egresadas, sin dejar de lado el papel preponderante que juega la planificación en los procesos de desarrollo.

\section{Conclusiones}

A continuación, se procede a enumerar las conclusiones:

1. El papel de la investigación en los procesos de planificación consiste en establecer relaciones analíticas sobre los problemas empíricos, que posteriormente se operacionalizarán en una propuesta concreta de intervención. En este sentido, los criterios de rigurosidad, pertinencia, sistematicidad y verificabilidad de los métodos científicos, así como las posturas teóricas sobre los objetos de planificación deben ser claramente expuestos en el diseño de un TFG, aspecto que no ha sido claro. Esto último es válido para cualquier maestría, sea esta académica o profesional o sea de cualquier otra disciplina.

2. Desde una perspectiva generalizada se ha considerado que la visión de una maestría profesional consiste en la aplicación de herramientas técnicas o únicamente para la formalización o diseño de determinadas propuestas de proyectos, prospección, coordinación, implementación o evaluación. En el caso de la maestría en Planificación, esto ha sido considerado lo normal. Por el contrario, la visión de la MP consiste en la capacidad que tiene el estudiantado de desarrollar conocimientos científicos sobre un problema empírico de la planificación. La utilización de herramientas técnicas de planificación deberá ser justificada teóricamente luego de haber detallado los contextos de la investigación. Ello por cuanto el diseño de la propuesta no solo es inherente a la planificación, sino que representa el aporte de la persona investigadora. una vez comprendido el problema estudiado. Este último componente se considera parte integral del trabajo final de investigación en la maestría profesional y principalmente en el caso de la maestría en Planificación. 
3. Un error frecuente ha sido que el estudiantado formule primero la propuesta de intervención, antes de saber si esa propuesta es viable y factible. En este abordaje existe una disonancia epistémica en donde el diagnóstico es solo la forma de justificar la propuesta preconcebida y no como debería ser: una forma de investigación científica que brinda la posibilidad de encontrar propuestas de intervención desde la participación, la intersubjetividad y el conocimiento profundo de los contextos socioeconómicos y culturales.

4. Entonces, el diagnóstico se constituye como un pertinente método debido a su capacidad heurística para establecer el puente entre la construcción de conocimiento científico sobre un objeto de estudio y la elaboración de propuestas de intervención desde la planificación. La relación entre diagnóstico y propuesta debe ser abordada como una unidad dentro de un proceso de planificación que considera que la segunda existe gracias a la primera.

5. No cabe duda de que la maestría profesional está pensada para enfatizar en generar y desarrollar conocimientos y habilidades disciplinares y, por tanto, la propuesta de planificación tiene una significancia mayor en el TFG. Sin embargo, el TFG debe estar sustentada en el análisis crítico y analítico del problema que origina la propuesta, sobre todo si el mismo representa la confluencia de variables complejas que ameritan abordajes multi e interdisciplinares.

6. Finalmente, según los criterios de este artículo, se concluye que es totalmente pertinente la existencia de las maestrías profesionales y por lo tanto las maestrías en Planificación, dado que estas generan capacidades no solo en investigación de problemas complejos y diversos, sino en el diseño de estrategias para su transformación. Así, la investigación y la propuesta solo tienen sentido de existencia en cuanto ambas se complementan. 


\section{Referencias}

Ander-Egg, E. (1991). Introducción a la planificación. Siglo XXI.

Ander-Egg, E \& Aguilar-Idáñez, J. (2013). Diagnóstico social. Conceptos y metodología. (Segunda ed.). Grupo editorial Lumen. Hvmanitas.

Arias, F. (2012). El Proyecto de Investigación. Introducción a la metodología científica. EDITORIAL EPISTEME.

Brenes-Chacón, A. (1987). Los Trabajos finales de Graduación: su elaboración y presentación en las ciencias sociales. EUNED.

Cerda, H. (1991). Los elementos de la investigación. El Búho.

Cervo, A. \& Bervian, P. (1989). Metodología científica. McGraw-Hill.

Consejo Nacional de Rectores [CONARE] (2012). Compendio Leyes, decretos y convenios de la educación superior universitaria estatal. Oficina de Planificación de la Educación Superior.

Delgado, G. (2010). Conceptos y metodología de la investigación histórica. Revista Cubana de Salud Pública, 36 (1), 9-18.

Hernández, A. G., Saavedra, J. J., \& Sanabria, M. (2006). La formación administrativa en Colombia: el caso de las maestrías. Revista Facultad De Ciencias Económicas, 14(2), 21-38. Recuperado de: https://revistas.unimilitar.edu.co/index.php/rfce/article/view/4572

Lira, L. (2006). Revalorización de la Planificación del Desarrollo. Instituto Latinoamericano y del Caribe de Planificación Económica y Social (ILPES). Publicación de las Naciones Unidas.

Lozada, J. (2014). Investigación Aplicada: Definición, Propiedad Intelectual e Industria. CienciAmérica. Revista de divulgación científica de la Universidad Tecnológica Indoamérica, 3 (1), 47-50.

Martín, J. (2005). Funciones básicas de la planificación económica y social. CEPAL. 
Máttar, J., \& Cuervo-González, L. (2017). Planificación para el desarrollo en América Latina y el Caribe: enfoques, experiencias y perspectivas. CEPAL.

MATUS, C. (1987). Política, planificación y gobierno. Segundo borrador.

Marco de Cualificaciones para la Educación Superior Centroamericana [MCESCA]. (2018). Resultados de aprendizaje esperados para los niveles técnico superior universitario, bachillerato universitario, licenciatura, maestría y doctorado. Editorial Serviprensa, Consejo Superior Universitario Centroamericano (CSUCA)

Nato, A. \& Carbajal, L. (2011). La conflictividad social desborda el dispositivo jurídico/judicial. Hacia un modelo de intervención integral en conflictos sociales urbanos o territoriales. Revista Jurídica Mario Alario D'Filippo, 3 (2), 31-45

Programa Estado de la Nación. (2017). Sexto informe estado de la educación. PEN.

Ríos, R. \& Quiroz-Bañol, J. (2018). Aproximación a un concepto de objeto de estudio disciplinar y a sus elementos constitutivos [Artículo de presentación]. XI Encuentro de la Asociación de Educación e Investigación en Ciencia de la Información de Iberoamérica y el Caribe, Medellín, Colombia.

Sánchez-Buitrago, J. (2009). Un concepto emergente de planeación. Clío América, 3(5) 39-59.

Torres-Carrillo, A. \& Jiménez-Becerra, A. (2004). La construcción del objeto y los referentes teóricos en la investigación social. UPN, Universidad Pedagógica Nacional. 
